\title{
Response surface-based finite element model calibration of a one-span historical masonry bridge
}

\author{
Emre Alpaslan*iD, Zeki Karaca (iD \\ Ondokuz Mayls University, Civil Engineering Department, Samsun, Turkey
}

\begin{abstract}
This study aims to investigate the dynamic behavior of a one-span historical masonry arch bridge. For this reason, the masonry bridge with $15.5 \mathrm{~m}$ in length and $4.75 \mathrm{~m}$ in width was chosen and the modal parameters were obtained by performing numerical analyses and experimental measurements. Operational Modal Analysis technique is utilized for experimental study to determine modal parameters of the historical masonry bridge. Sensitive three-axial accelerometers were located on critical points on the bridge span and signals originated by accelerometers were collected to quantify the vibratory response of the historical bridge. The Enhanced Frequency Domain Decomposition and Stochastic Subspace Identification method are employed to identify the natural frequencies, mode shapes, and damping ratios experimentally. The 3D finite element modeling of the historical masonry bridge was created and the natural frequencies and mode shapes of the bridge were determined numerically. Experimental results were compared with those of the finite element analysis of the bridge. It can be noticed significant differences when comparing the results of the experimental and numerical with the initial conditions. Therefore, the finite element model is calibrated by using the response surface method according to the experimental results to minimize the uncertain finite element modeling parameters of the historical masonry arc bridge such as material properties and boundary conditions. At the end of the calibration process, the maximum differences between natural frequencies obtained experimental measurements and numerical analysis decreases from 4\%-50\% to $0.5 \%-2.5 \%$. The final calibrated finite element model for the masonry bridge is able to produce natural frequencies in close agreement with the measured ones.
\end{abstract}

\section{Keywords}

Response surface model; Model calibration; Masonry bridge; Finite element model.

Received: 13 December 2019; Accepted: 19 February 2020

ISSN: 2630-5763 (online) C) 2020 Golden Light Publishing All rights reserved.

\section{Introduction}

In general, all engineering fields form numerical or mathematical models in order to obtain the behavior of real systems. These numerical models may cover a wide range of purposes. But they can be classified under three main headings; analysis, forecast, and design. In civil engineering, Finite element (FE) models are one of the most common methods used to obtain the responses of structures under dynamic loads such as earthquakes, wind, traffic, and explosion. These FE analyses can also be utilized for the design of part or all of the structure. There is a need to examine the validity of the established numerical modeling for all purposes mentioned herein. Because while creating numerical modeling, assumptions about many unknown and

* Corresponding author

Email: emre.alpaslan@omu.edu.tr 
uncertain system parameters such as geometrical and material properties, loading and boundary conditions must be made. In addition to these, due to lack of information or some limitations, it is necessary to simplify the assumptions used in the construction of structures. For these reasons, the accuracy and validity of the numerical models can be reduced.

For the reasons mentioned above, model calibration techniques have been developed and named as a model calibration or, more clearly, parameter estimation or determination. In general terms, the model calibration technique aims to update or re-evaluate the unknown structural system properties used as a parameter in numerical modeling. The data (acceleration-time values, frequency response functions, natural frequencies, and natural mode shapes, modal stresses and curvature grades, modal elasticity) obtained from the vibration experiments give detailed information about the general and local behaviors of the structure. Therefore, this vibration data has an important role in the FE model calibration process.

Response surface (RS) methodology is a combination of statistical, and mathematical techniques to represent the relationship between the inputs and outputs of a physical system by explicit functions. This methodology has been widely employed in many applications such as design optimization, response prediction, and model validation. The model calibration techniques have been applied in many civil engineering structures such as bridges, dams, masonry structures, steel frame structures and reinforced concrete structures [1-6].

Historic masonry arch bridges with different shapes, sizes and openings are among the most important historical structures. Because of the social, economic and strategic importance of such structures, more studies are needed to understand their needs for conservation, restoration, and strengthening. Therefore, it is important to understand the modal parameters of such historical structures more realistically. This study is based on a part of the doctoral dissertation by Alpaslan [7] and aims to reveal that the RS method is a suitable and correct method for model calibration in masonry arch bridge structures. For this purpose, the Operational Modal Analysis (OMA) technique for the field study is used to obtain modal parameters of the Sarpdere Historic Masonry Arch Bridge in Ordu, Turkey and the values of the structural parameters obtained from the field measurements are compared with the values identified by the FE model. By using the RS method, the natural frequency values corresponding to the first three mode shapes of the structure is tried to be converged with the frequency values identified by the experimental measurements.

\section{Material and method}

\subsection{Historical sarpdere masonry bridge}

The Historical Sarpdere Bridge is located on the Ordu-Ulubey road, $33 \mathrm{~km}$ from Ordu and $9 \mathrm{~km}$ from Ulubey, in the Şihlar village, on the Sarpderesi. The bridge was built as a single-span bridge in the 1870s during the Ottoman Empire and became one of the most important bridges between Ordu and Sivas. In time, it was aimed to preserve this historical culture due to the deterioration of the material properties and boundary conditions of the historical arch bridge. In 2012, the restoration work was completed by the 7th Regional Directorate of Samsun Highways (Fig. 1). The historic Sarpdere Bridge is an arch bridge built of natural stone. The bridge is $15.50 \mathrm{~m}$ long and $4.75 \mathrm{~m}$ wide. The bridge is composed of the arch part which has four different radii, sidewalls, filling material, walkway, and railing. The geometric features of the historical bridge are shown in Fig. 2. Horasan mortar is used as the binding material. Limestone was used in bridge construction. The mechanical properties of the materials used for the restoration process are shown in Table 1 [8]. The material properties of the Sarpdere Bridge are similar to those of this type of bridge studied in the literature [9].

The flowchart of the study is summarized in Fig. 3. The first step is that the initial finite element model of the bridge is constructed by using the material and geometrical properties and modal analysis i performed to investigate the dynamic behavior of the historic Sarpdere Bridge. 


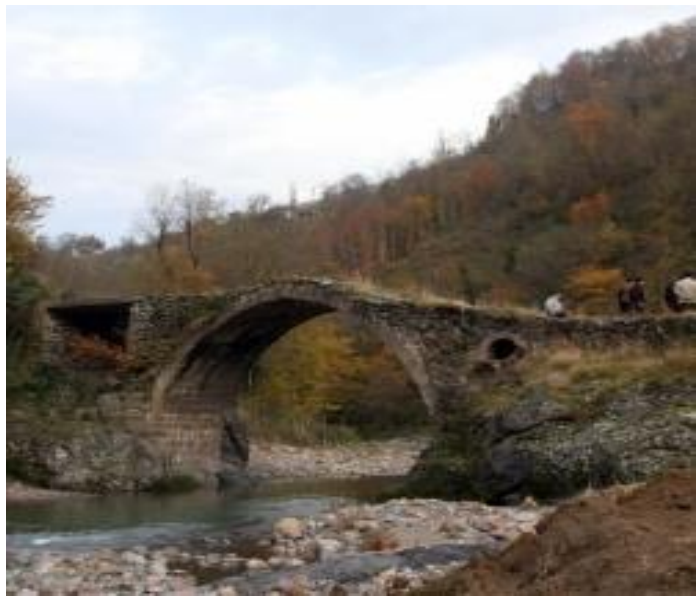

(a)

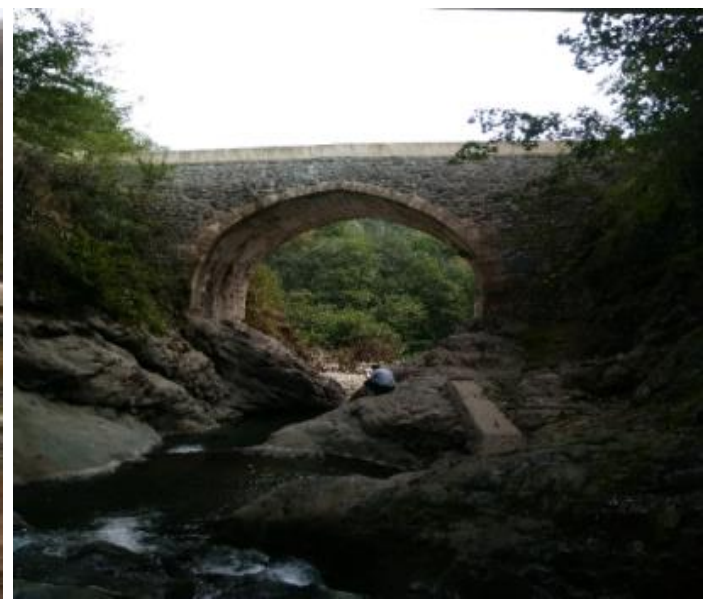

(b)

Fig. 1. Historical Sarpdere Bridge (a) before restoration (b) after Restoration

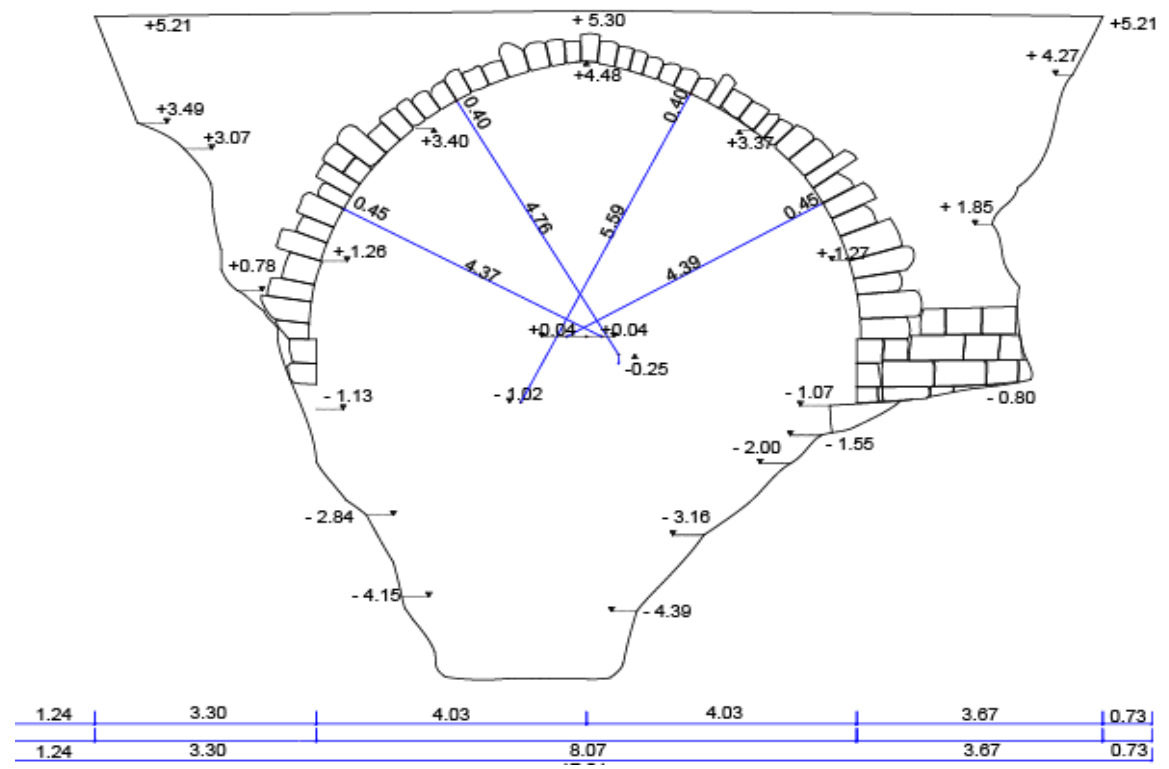

(a)

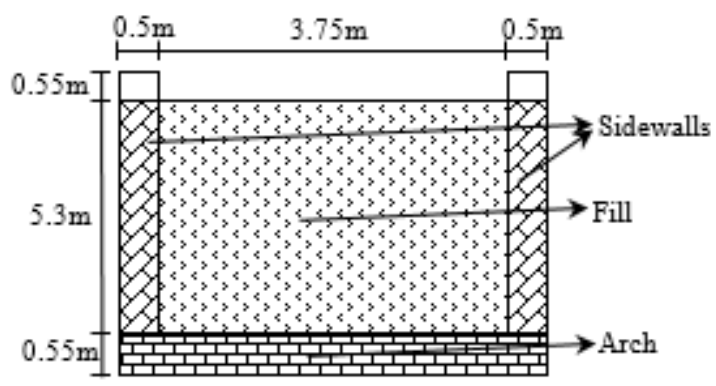

(b)

Fig. 2. Geometrical properties of Historical Sarpdere Bridge (a) front view (b) side view 
Table 1. Material Properties of Sarpdere Bridge

\begin{tabular}{lccc}
\hline & Unit Weight $\left(\mathrm{kg} / \mathrm{m}^{3}\right)$ & Modulus of Elasticity (MPa) & Poisson Ratio \\
\hline Arch & 1900 & 3000 & 0.2 \\
Side walls & 1800 & 2500 & 0.2 \\
Slab & 1800 & 2500 & 0.2 \\
Filling material & 1365 & 1500 & 0.05 \\
\hline
\end{tabular}

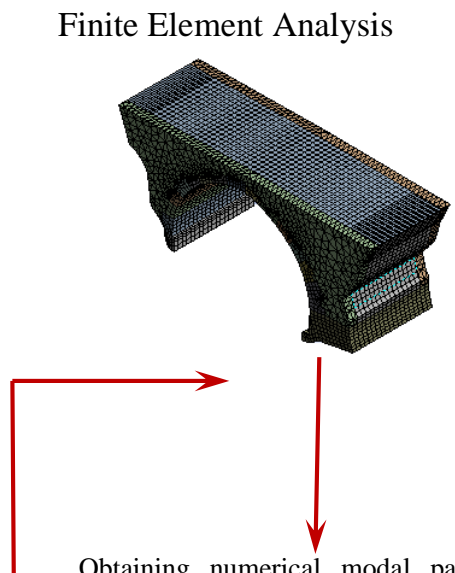

Obtaining numerical modal parameters (natural frequencies and mode shapes)
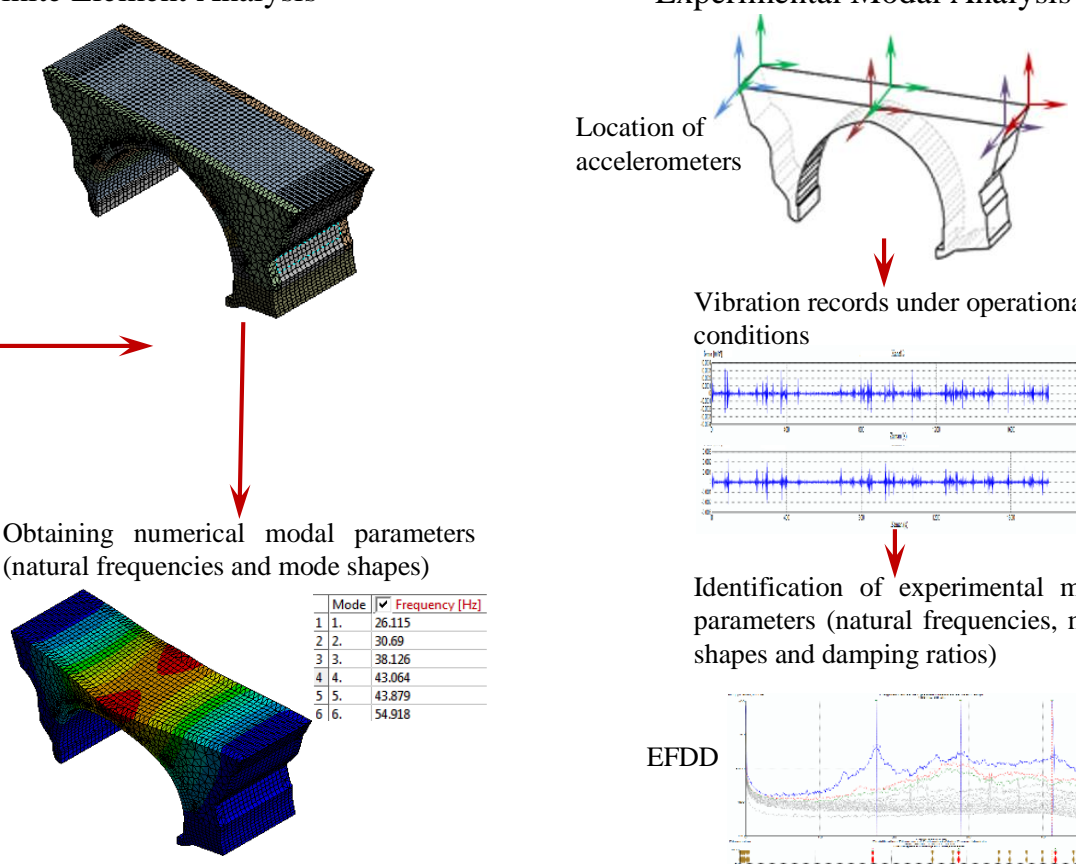

Vibration records under operational conditions

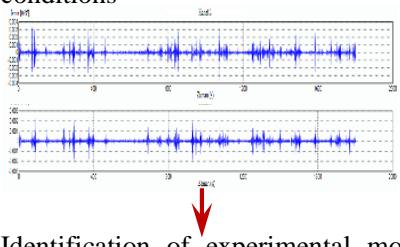

Identification of experimental modal parameters (natural frequencies, mode shapes and damping ratios)

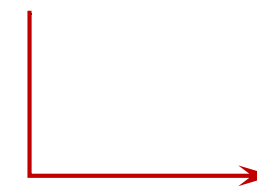

Comparison of experimental and numerical modal parameters<smiles>C1CC2CC1C2</smiles>

Error $\leq \% 5$

FE Model Calibration

RS method

-Design of experiment

-Response surface

-Optimization

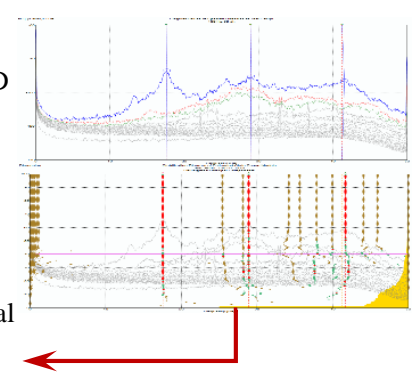

EFDD

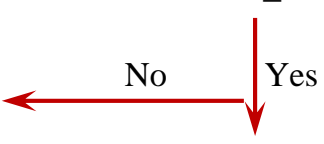

Ideal FE Model

Fig. 3. Flowchart of the study

Then, experimental studies are conducted on the bridge considering the natural frequency values obtained from the numerical analysis. In the experimental study, the vibrational response of the bridge is recorded under environmental conditions by using the operational modal analysis method. Natural frequencies, mode shapes and damping ratios of the bridge are identified by using the 
vibration records. Finally, the initial FE model was calibrated according to the obtained natural frequency values experimentally. In this process, it was tried to represent the dynamic behavior of the historical bridge more realistically.

\subsection{Enhanced frequency domain decomposition}

In general, the Enhanced Frequency Domain Decomposition (EFDD) technique is used for OMA in the civil engineering industries. In the EFFD technique, the spectral density matrix is approximately separated into a set of single degree of freedom (SDOF) systems utilizing the Singular Value Decomposition. It is possible to get exact results in the case where loading is white noise, the structure is lightly damped, and if the mode shapes of close modes are geometrically orthogonal. Even if these assumptions are not satisfied, the results are significantly reasonable. The relationship between unknown input $\mathrm{x}(\mathrm{t})$ and the measured responses $\mathrm{y}(\mathrm{t})$ is expressed as $[10,11]$

$$
G_{y y}(j w)=H(j w)^{*} G_{x x}(j w) H(j w)^{T}
$$

in which $G_{x x}$ is the Power Spectral Density (PSD) matrix of the input signal, $G_{y y}$ is the PSD matrix of the output signal, $H(j \omega)$ is the Frequency Response Function (FRF) matrix, and $*$ and $T$ describe complex conjugate and transpose, respectively. The FRF matrix $H(j \omega)$ is defined as

$$
H(j w)=\sum_{k=1}^{n} \frac{R_{k}}{j w-\lambda_{k}}+\frac{R_{k}^{*}}{j w-\lambda_{k}^{*}}
$$

in which $n$ is mode number, $\lambda_{k}$ is the pole and $R_{k}$ is the residue matrix. Using Eq. (2) into Eq. (1), it is obtained [12] as

$$
\begin{aligned}
G_{y y}(j w) & =\sum_{k=1}^{n} \sum_{s=1}^{n}\left[\frac{R_{k}}{j w-\lambda_{k}}+\frac{R_{k}^{*}}{j w-\lambda_{k}^{*}}\right] \\
& \times G_{x x}(j w)\left[\frac{R_{s}}{j w-\lambda_{s}}+\frac{R_{s}^{*}}{j w-\lambda_{s}^{*}}\right]^{\bar{H}}
\end{aligned}
$$

in which $s$ is singular values and $\bar{H}$ is complex conjugate and transpose. After some mathematical handling, the output PSD can be reduced to a pole/residue form as follows

$$
\begin{aligned}
G_{y y}(j w) & =\sum_{k=1}^{n} \frac{A_{k}}{j w-\lambda_{k}}+\frac{A_{k}^{*}}{j w-\lambda_{k}^{*}} \\
& +\frac{B_{k}}{-j w-\lambda_{k}}+\frac{B_{k}^{*}}{-j w-\lambda_{k}^{*}}
\end{aligned}
$$

This is the pole-residue form of the output PSD matrix. $A_{k}$ is the $k$-th residue matrices of the output PSD; it is a $1 \times 1$ Hermitian matrix given by

$$
\left[A_{k}\right]=\left[R_{k}\right][C]\left(\sum_{s=1}^{n} \frac{\left[R_{s}^{H}\right]}{-\lambda_{k}-\lambda_{s}^{*}}+\frac{\left[R_{s}^{T}\right]}{-\lambda_{k}-\lambda_{s}}\right)
$$

When just the k-th mode is considered, the following contribution can be obtained as;

$$
\left[A_{k}\right]=\frac{\left[R_{k}\right][C]\left[R_{k}\right]^{H}}{2 \sigma_{k}}
$$

When the damping is light, this term can become dominating, thus obtaining a residue which is proportional to the mode shape vector as follows:

$$
\begin{aligned}
{\left[A_{k}\right] \infty\left[R_{k}\right]\left[C\left[R_{k}\right]^{H}\right] } & =\{\varphi\}_{k}\{\gamma\}_{k}^{T}[C]\{\gamma\}_{k}\{\varphi\}_{k}^{T} \\
& =d_{k}\{\varphi\}_{k}\{\varphi\}_{k}^{T}
\end{aligned}
$$

where $d_{k}$ is a scaling factor for the $\mathrm{k}$-th mode.

Considering a lightly damped system and that the contribution of the modes at a particular frequency is limited to a finite number, then the response spectral density matrix can be written in the following final form;

$$
\left[G_{y y}(w)\right]=\sum_{k \in S u b(\omega)} \frac{d_{k}\{\varphi\}_{k}\{\varphi\}_{k}^{T}}{j \omega-\lambda_{k}}+\frac{d_{k}^{*}\{\varphi\}_{k}^{*}\{\varphi\}_{k}^{* T}}{j \omega-\lambda_{k}^{*}}
$$

where $k \in \operatorname{Sub}(\omega)$ is the set of modes that contribute at the considered frequency. Performing the singular value decomposition of the output PSD matrix known at discrete frequencies $\omega=\omega_{i}$ one obtains

$$
G_{y y}\left(j w_{i}\right)=U_{i} S_{i} U_{i}^{\bar{H}}\{U(t)\}
$$

in which the matrix $U_{i}$ represents a unitary matrix holding the singular vector $u_{i j}, S_{i}$ is the diagonal 
matrix holding the scalar singular values $s_{i j}$. PSD matrix in Eq. (9), peak values and singular vectors, $u_{i j}$, are corresponds to natural frequencies and natural mode shapes, respectively.

\subsection{Stochastic subspace identification}

The Stochastic Subspace Identification (SSI) technique, in contrast to the EFDD technique, uses time-domain techniques, without the need to convert them to correlations and spectra. The dealing directly with raw response time series, data order, and deterministic input signal, noise reduced by orthogonal projection and synthesis from decomposition are some main characteristics of the SSI technique. The technique SSI has firstly introduced by Van Overschee and De Moor [13] and then developed by several authors [14, 15]

The SSI method has some major benefits as follows. First, the SSI techniques work in the time domain and are data-driven methods. Since the model estimation is not relying on any Fourier transformations to frequency domain no leakage is introduced. Second, the SSI algorithms are born linear least-squares fitting techniques fitting state space systems with correct noise modeling. This leads to the use of much smaller model orders than other commercially available high order model estimators. Third, all SSI techniques include automatic mode estimation that searches the stable modes in the SSI Stabilization Diagram. All stable modes of all estimated models of all test setups are included in the search, and the result is modal estimates of natural frequencies, damping ratios and modes shapes of high accuracy.

Modal parameters of the structure can be expressed by a set of linear, constant coefficients and second-order differential equations:

$$
\begin{array}{r}
{[M]\{\ddot{U}(t)\}+[C]\{\dot{U}(t)\}+[K]\{U(t)\}} \\
=\{R(t)\}=[B](u(t))
\end{array}
$$

where $[M],[C]$ and $[K]$ are the mass, damping and stiffness matrices, respectively, $\{R(t)\}$ is the excitation force, $\{\ddot{U}(t)\}, \quad\{\dot{U}(t)\}$ and are acceleration, damping and displacement vectors at continuous time $t$, respectively. The force vector can be described in a matrix $[B]$ defined the inputs in space and a vector $u(t)$. By using the following definitions

$$
\begin{aligned}
& x(t)=\left(\begin{array}{c}
U(t) \\
\dot{U}(t)
\end{array}\right), \quad A_{*}=\left(\begin{array}{cc}
0 & I \\
-M^{-1} K & -M^{-1} C
\end{array}\right), \\
& B_{*}=\left(\begin{array}{c}
0 \\
M^{-1} B
\end{array}\right)
\end{aligned}
$$

Eq. (11) can be written into the state equation;

$$
\dot{x}(t)=A_{*} x(t)+B_{*} u(t)
$$

where $A_{*}$ is the state matrix, $B_{*}$ is the input matrix and $x(t)$ is the state vector. The number of elements of the state-space vector is the number of independent variables needed to describe the state of a system. If it is assumed that the measurements are evaluated at only one sensor locations and that these sensors can be accelerometers, velocity or displacement transducers, the observation equation is

$$
y(t)=C_{a} \ddot{U}(t)+C_{u} \dot{U}(t)+C_{d} U(t)
$$

where $y(t)$ are the outputs, and $C_{a}, C v, C_{d}$, are the output matrices for displacement, velocity, acceleration. With the following definitions,

$$
\left.\begin{array}{c}
C=\left[\begin{array}{cc}
C_{d}-C_{a} M^{-1} K & C_{v}-C_{a} M^{-1} C_{*}
\end{array}\right] \\
D=C_{a} M^{-1} B_{*}
\end{array}\right\}
$$

Eq. (10) can be transformed into:

$$
y(t)=C x(t)+D u(t)
$$

where $C$ is the output matrix and $D$ is the direct transmission matrix. Eqs (12) and (15) constitute a continuous-time deterministic state-space model. Continuous-time means that the expressions can be evaluated at each time instant $t \in R$ and deterministic means that the input-output quantities $u(t), y(t)$ can be measured exactly. Of course, this is not realistic: measurements are available at discrete time instants $k \Delta t, k \in N$ with $\Delta t$, the sample time and noise are always influencing the 
data. After sampling, the state-space model looks like

$$
\left.\begin{array}{c}
x_{k+1}=A x_{k}+B u_{k} \\
y_{k}=C x_{k}+D u_{k}
\end{array}\right\}
$$

where $x_{k}=x(k \Delta t)$ is the discrete-time state vector, $A=\exp \left(A_{c} \Delta t\right)$ is the discrete state matrix and $B=[A-I] A_{c}^{-1} B_{c}$ is the discrete input matrix. If $A_{c}$ is not invertible, another expression holds for $B$. The stochastic components (noise) are included and we obtain the following discrete-time combined deterministic-stochastic state-space model:

$$
\left.\begin{array}{c}
x_{k+1}=A x_{k}+B u_{k}+\omega_{k} \\
y_{k}=C x_{k}+D u_{k}+v_{k}
\end{array}\right\}
$$

where $\omega_{k}$ is the process noise due to disturbances and modeling inaccuracies and $v_{k}$ is the measurement noise due to sensor inaccuracy. They are both immeasurable vector signals but we assume that they are zero-mean, white and with covariance matrices:

$$
E\left[\left(\begin{array}{l}
\omega_{p} \\
v_{p}
\end{array}\right) \quad\left(\begin{array}{ll}
\omega_{q}^{T} & v_{q}^{T}
\end{array}\right)\right]=\left(\begin{array}{cc}
Q & S \\
S^{T} & R
\end{array}\right) \delta_{p q}
$$

where $E$ is the expected value operator and $\delta_{p q}$ is the Kronecker delta.

The vibration information that is available in structural health monitoring is usually the responses of a structure excited by the operational inputs that are some immeasurable inputs. Due to the lack of input information, it is impossible to distinguish deterministic input $u_{k}$ from the noise terms $\omega_{k}, v_{k}$ in Eq. (17). If the deterministic input term $u_{k}$ is modeled by the noise terms $\omega_{k}, v_{k}$ the discrete-time purely stochastic state-space model of a vibration structure is obtained:

$$
\left.\begin{array}{l}
x_{k+1}=A+\omega_{k} \\
y_{k}=C x_{k}+v_{k}
\end{array}\right\}
$$

in which $x_{k}$ is the Kalman sequences that are placed in SSI by a so-called orthogonal-projection technique, $y_{k}$ is the outputs. $A$ and $C$ are obtained from regression Eq. (19). $\omega_{k}$ represents the process noise due to disturbances and modeling inaccuracies and $v_{k}$ is the measurement noise due to sensor inaccuracy. Eq. (19) presents the general form of the time-domain system identification through operational vibration measurements. Comprehensive explanations about the equations can be found in the literature [13].

\subsection{Modal assurance criterion (MAC)}

Modal Assurance Criterion (MAC) can be used in modal analysis applications due to its capability to compare different mode shapes in one number. The value of MAC is between 0 and 1 . If there is enough agreement between mode shapes, the MAC values are around 1. For two different mode shapes $\left\{\phi_{A}\right\},\left\{\phi_{X}\right\}$, MAC can be calculated as follows [16]:

$$
\operatorname{MAC}(r, s)=\frac{\left|\left\{\phi_{A}\right\}_{r}^{T}\left\{\phi_{X}\right\}_{s}\right|^{2}}{\left(\left\{\phi_{A}\right\}_{r}^{T}\left\{\phi_{A}\right\}_{r}\right)\left(\left\{\phi_{X}\right\}_{s}^{T}\left\{\phi_{X}\right\}_{s}\right)}
$$

where $\left\{\phi_{X}\right\}_{s}$ is test modal vector, mode $s,\left\{\phi_{A}\right\}_{r}$ is a compatible modal vector, mode $r$ and $T$ is the transpose.

\subsection{Response surface method}

Model calibration methods are divided into two groups as non-iterative and iterative methods. Noniterative methods directly update the stiffness and mass matrices of the numerical model through a closed-form direct solution. However, such methods cause the loss of structural connectivity, and the proposed corrections are not always physically meaningful. On the other hand, iterative methods require sensitivity matrices to conduct iteration in minimizing the objective function. However, the sensitivity-based method seems not practical in case of high degrees of freedom structural system, as it results in the problem of slow convergence and a time-consuming process due to the increase in the degree of freedom. Therefore, the RS method has appeared as an 
alternative tool in the FE model calibration process due to simplicity and provides fast optimization because of smooth gradients, thus decreasing the convergence problem [17].

RS method is an approximate optimization method that looks at various design variables and their responses, which seeks the best experimental design using the minimum number of design samples, to determine the combination of design variables. RSM can achieve a satisfactory accuracy between the measured data and the FE modelgenerated data $[18,19]$. As a consequence of this, RSM can carry out to replace the implicit functions of the original design optimization problem with an approximation model, which is usually simple and not computationally intensive as opposed to a full FE model. RSM method, therefore, is a very useful model to optimize the FE model for matching measured data to the FE model-generated data. The ANSYS DesignXplorer [20] used in this study employs the second-order approximation method in RS creation. Fig. 4 demonstrates the steps of the FE model calibration procedure. Firstly the calibrating parameters are selected and lower and upper limits are assigned to initial parameter values. Then, the response feature can be calculated by the FE analysis according to the design of experiments generated by the central composite design in this study. The next step is the construction and verification of the RS models. Finally, optimization can be conducted within the RS models, identified calibrated parameters can be transferred to the FE model.

\subsubsection{Design of experiments}

Design of experiments is a technique used to scientifically determine the location of sampling points. There are a variety of design of experiments algorithms or methods in engineering studies namely star, full factorial, central composite, and Box-Behnken designs.

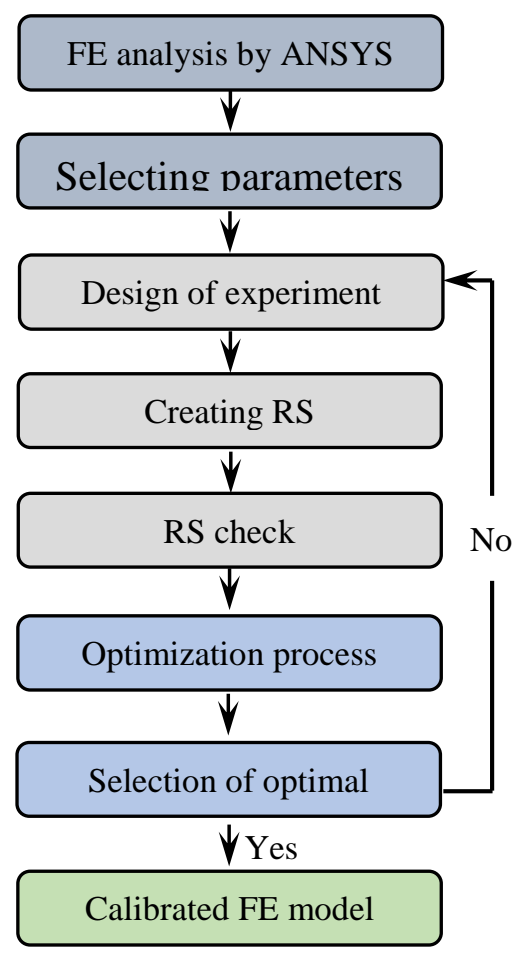

Fig. 4. Flowchart of response surface model calibration process

Sample points in effective locations not only reduce the required number of sampling points but also increase the accuracy of the response surface obtained from the results of the sampling points. In this study, the central composite design for three variables is selected as a sampling method.

\subsubsection{Generation of RS model}

In this study, a genetic aggregation technique was used to generate RS models. The technique automates the selection, configuration, and creation of the RS. It automatically generates the most suitable approach for each output and provides more reliable results than other RS models due to multiple solutions of RS and cross-validation processes. The fitted RS models should be used to conduct the FE model calibration after validated through their fitness. R2 criterion and root mean squared error (RMSE) criterion are usually utilized for multi-RSM and complicated models [21, 22]. Rsquare Statistic and Relative Mean Square Error are presented in Eq. (21) and Eq. (22) are regarded as 
the criteria to check the fitness of the fitted RS models.

$$
\begin{gathered}
R^{2}=1-\frac{\sum_{j=1}^{N}\left[y_{R S}(j)-y(j)\right]^{2}}{\sum_{j=1}^{N}[y(j)-\bar{y}]^{2}} \\
R M S E=\frac{1}{N^{*} \bar{y}} \sqrt{\sum\left[y_{R S}(j)-y(j)\right]^{2}}
\end{gathered}
$$

In which $y_{R S}$ describes the function value of the obtained RS model, $y$ is FE calculation results, $\overline{\mathrm{y}}$ is the mean of $y$; and $N$ defines the number of confirmation sample points in design space. The larger the value of $\mathrm{R}$ Square is, the more accurate the RS model. The smaller the value of RSME is, the more accurate the RS model.

\subsubsection{Optimization}

In the structural dynamic, the natural frequencies and mode shapes are crucial parameters for the structural response features. In this study, the natural frequency was taken as a response feature. The primary model previously obtained is calibrated herein to seek the reference values of input parameters by minimizing the response discrepancies between the primary RS model and the experimental model. The measured frequencies from the bridge employing OMA are used as the objective responses. Optimization computation in this study is carried out with the screening approach. This approach is a non-iterative direct sampling method by a quasi-random number generator based on the Hammersley algorithm. In general, the screening method is the most convenient way to carry out a preliminary design study, since it is a low-resolution, fast and detailed study that can be useful to quickly find approximate solutions [20].

\section{Results}

\subsection{Initial finite element model}

To obtain the natural frequency and mode shapes of the historical Sarpdere Bridge, the 3D finite element model was created by using the ANSYS Workbench [20] software program. The bridge model was consist of 5 parts, two side walls, one filling, one arch, and one floor. Body element was used for connection of the parts and solid186 threedimensional solid element model was chosen as an element type. The boundary conditions of the bridge model were assigned as fixed support to the right and left sides (Fig. 5). The transverse, bending and torsional mode shapes obtained from the bridge model and their corresponding natural frequencies are shown in Fig. 6.

\subsection{Experimental modal identification of sarpdere bridge}

Modal parameters of the historical bridge (natural frequency, mode shape, and damping ratio) were identified by using the OMA method. Ambient vibration signals under vehicle and wind loads were obtained by using a data acquisition system and two uniaxial and five triaxial accelerometers with a range of $0.1-120 \mathrm{~Hz}$ placed on 6 different locations at $4 \mathrm{~m}$ intervals on the bridge. Fig. 7 shows the experimental study. The measurement time and frequency range were selected as $30 \mathrm{~min}$ and 0-100 Hz. Vibration signals from accelerometers are recorded using Testlab_V2 software via the data acquisition system. The experimental modal parameters of the historical bridge were analyzed using ARTeMIS Modal 1.5 software [23].

Natural frequencies, mode shapes and damping ratios of the masonry bridge were obtained by using EFDD and SSI method as represented in Fig. 8. Mode shapes were identified as the transverse, bending and torsional mode. Also, experimentally obtained mode shapes of the masonry bridge are shown in Fig. 9.

The compatibility of the mode shapes obtained in both EFDD and SSI methods is examined with Modal Assurance Criteria (MAC) and the results are shown in Fig. 10. 


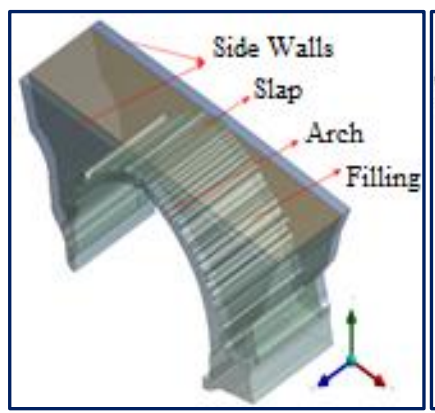

(a)

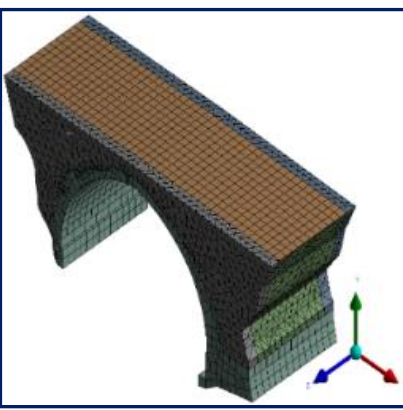

(b)

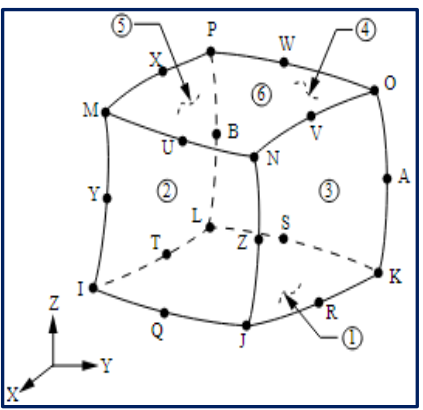

(c)

Fig. 5. Finite element model of the bridge (a) geometry, (b) finite element model, (c) solid186 element

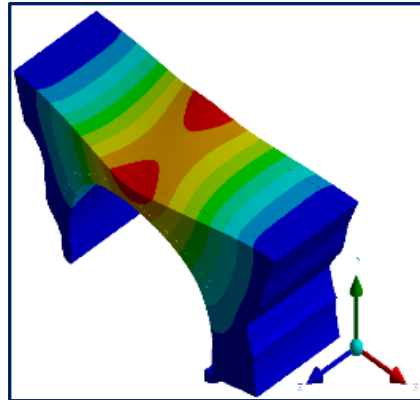

(a)

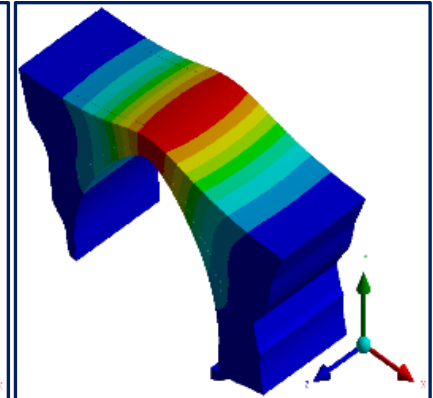

(b)

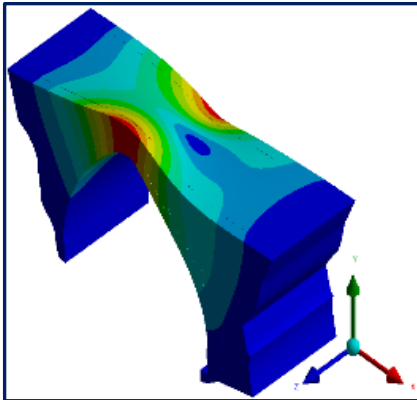

(c)

Fig. 6. Numerical identified mode shapes (a) transverse mode, $26.216 \mathrm{~Hz}$,

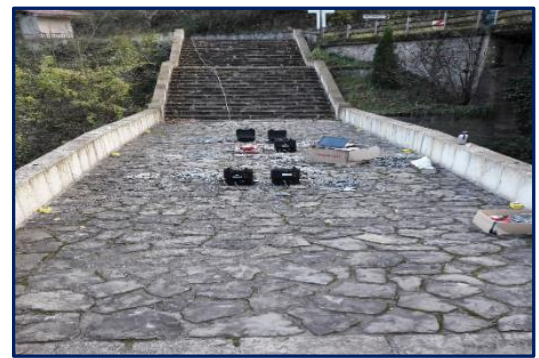

(a)

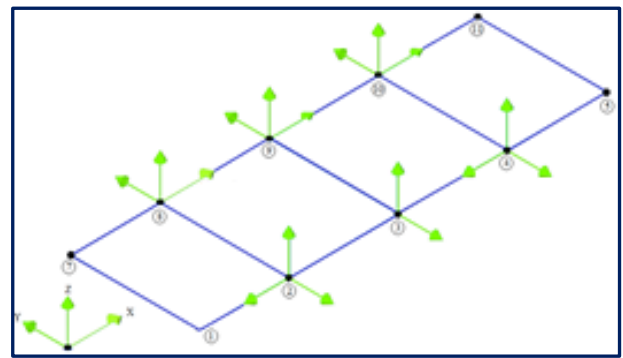

(b)

Fig. 7. Experimental study (a) test setup (b) accelerometer configurations

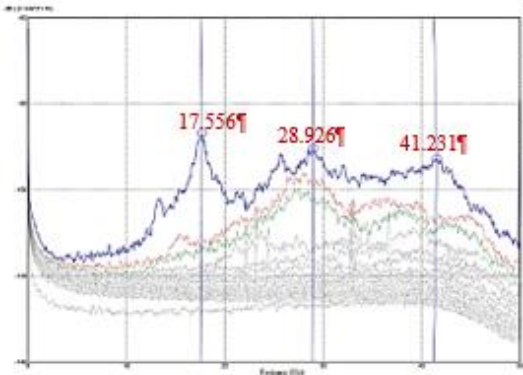

(a)

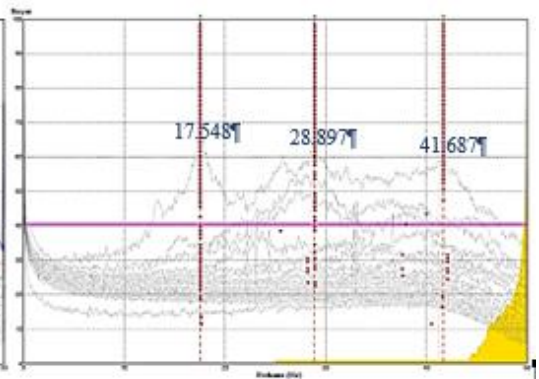

(b)

Fig. 8. Experimentally obtained natural frequencies (a) EFDD, (b) SSI 


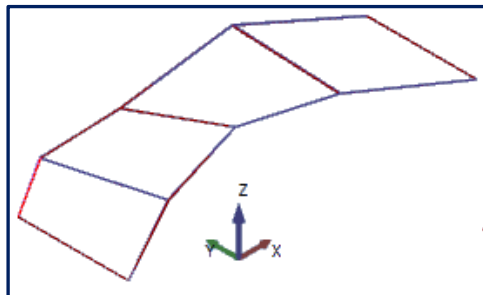

(a)

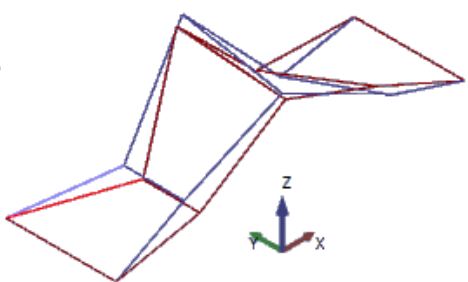

(b)

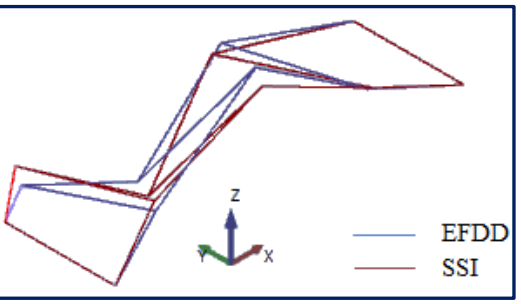

(c)

Fig. 9. Experimentally obtained mode shapes (a) transverse mode, (b) bending mode, (c) torsional mode

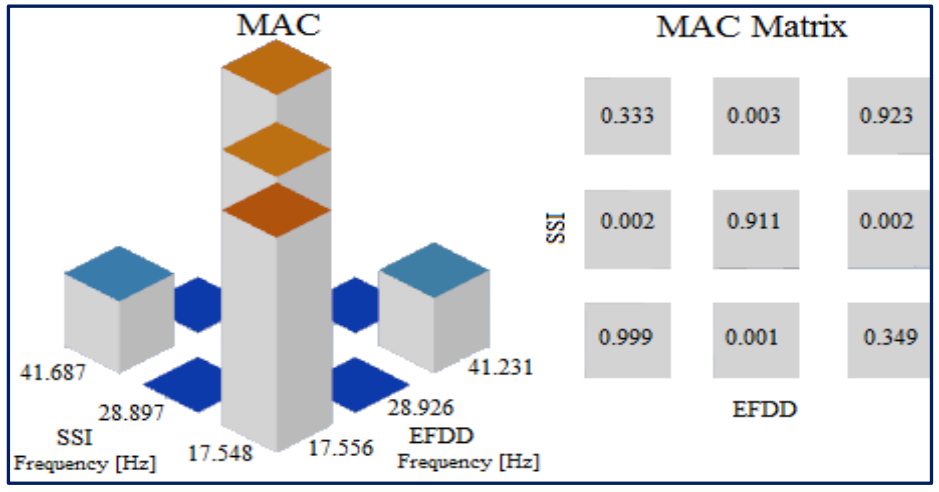

Fig. 10. MAC values

As can be understood from the experimental results, the harmony between both natural frequencies and mode shapes of the historical masonry bridge obtained by EFDD and SSI methods demonstrate that applied experimental study is appropriate to identify the dynamic behavior of the historical masonry bridge. It is also important to check the obtained natural frequency according to some empirical formulations related to the masonry type bridges. The study performed by Onat [24] represents different empirical formulations including some parameters such as unit weight, elasticity modulus of masonry materials, length, height, width and main span length of the masonry bridge. It can be understood that the empirical formulation consisting of both mechanical and physical quantities gives a more reliable result when compared to those with the natural frequencies obtained experimentally.

Table 2 represents the comparison between experimental and numerical natural frequencies. It appears that significant differences have occurred between natural frequencies obtained by numerically and experimentally, especially in transverse mode. Therefore, the initial FE model needs to be calibrated to minimize the differences and to create a more realistic FE mode that could represent the dynamic behavior of the historical bridge more accurately.

\subsection{Finite element model calibration}

For the FE model calibration process, natural frequencies evaluated from the modal analysis of the FE model are compared with the experimental measurements and the optimal results are found by using the optimization method. This method aims to close the numerical results to the experimental ones. For this reason, a total of 8 solid components was assigned to the right and left sides in the FE model of the masonry bridge to reflect the nature of the real bridge conditions as seen in Fig. 11. The frictional connection was used between solid components and structural components. The coefficient of friction was chosen as 0.01 . The boundary conditions of components 1 and 5 are assigned as fix support for both backside and bottom surfaces. 
Table 2. Comparison of natural frequencies

\begin{tabular}{cccccccc}
\hline \multicolumn{2}{c}{$\begin{array}{c}\text { Experimental } \\
\text { Mod }\end{array}$} & $\begin{array}{c}\text { Natural frequencies, } \\
f(\mathrm{~Hz})\end{array}$ & $\begin{array}{c}\text { Experimental } \\
\text { Damping Ratios, } \\
\xi(\%)\end{array}$ & $\begin{array}{c}\text { Numerical natural } \\
\text { frequencies with } \\
\text { initial } \\
\text { conditions, } f(\mathrm{~Hz})\end{array}$ & Error $(\%)$ \\
\hline & GFTAA & SAB & GFTAA & SAB & & GFTAA & SAB \\
1 & 17.56 & 17.55 & 2.326 & 3.605 & 26.22 & 49.32 & 49.40 \\
2 & 28.93 & 28.90 & 1.157 & 3.063 & 30.66 & 5.98 & 6.09 \\
3 & 41.23 & 41.69 & 0.560 & 3.228 & 42.86 & 3.95 & 2.81 \\
\hline
\end{tabular}

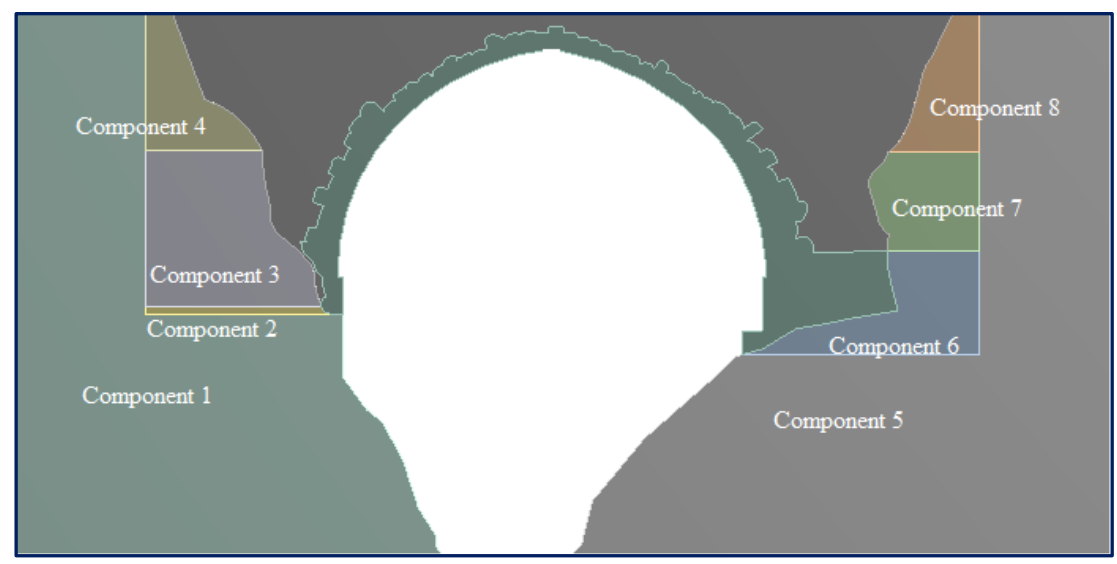

Fig. 11. Solid elements assigned as abutments

The material properties (modulus of elasticity, unit weight, and Poisson ratio) used for the solid components for the FE analysis are presented in Table 3. The modulus of elasticity of components 1 and 5 was chosen as the high value to satisfy the infinite rigidity of the axial plane of the bridge model. By performing a few trials and error FE analysis, it was decided that solid components should be formed according to low ground class rocks [25].

\subsubsection{Design of experiments}

The modulus of elasticity values of the solid components was used as calibrating parameters. The lower and upper values of the modulus of elasticity of the solid components are demonstrated in Table 4. A total of 45 design points was constructed and computed. Natural frequencies of the transverse, bending and torsional modes are selected as the output parameters and the parameters' name is $\mathrm{P} 7, \mathrm{P} 8, \mathrm{P}$, respectively.

\subsubsection{Responses surfaces}

The accuracy of the generated RS models is checked by using R2 and root mean squared error (RMSE) criteria. R2 and RMSE values obtained for each corresponding mode are shown in Table 5. It is demonstrated that all R2 and RMSE values are close to 1 and 0 , respectively, which represents that the obtained RS models have a high regression accuracy.

\subsubsection{Optimization}

After the RS models are obtained, the natural frequency values corresponding to the three mode shapes is tried to be converged with the frequency values identified by the experimental measurements. For this purpose, experimentally obtained natural frequencies are used as objective values as demonstrated in Fig. 12. The candidate point is determined in the optimization process. 
Table 3. Material properties of the solid components

\begin{tabular}{cccc}
\hline Component & Modulus of elasticity $(\mathrm{MPa})$ & Unit weight $\left(\mathrm{kg} / \mathrm{m}^{3}\right)$ & Poisson ratio \\
\hline 1 & $1 \times 10^{9}$ & 2000 & 0.2 \\
2 & 4000 & 2000 & 0.2 \\
3 & 2000 & 2000 & 0.2 \\
4 & 2000 & 2000 & 0.2 \\
5 & $1 \times 10^{9}$ & 2000 & 0.2 \\
6 & 4000 & 2000 & 0.2 \\
7 & 4000 & 2000 & 0.2 \\
8 & 2000 & 2000 & 0.2 \\
\hline
\end{tabular}

Table 4. Upper and lower limits of the modulus of elasticity of components

\begin{tabular}{lcccccc}
\hline Component No & 2 & 3 & 4 & 6 & 7 & 8 \\
Parameter No & P1 & P2 & P3 & P4 & P5 & P6 \\
Lower limit (MPa) & 2000 & 1000 & 1000 & 2000 & 2000 & 1000 \\
Upper limit (MPa) & 6000 & 4000 & 4000 & 6000 & 6000 & 4000 \\
\hline
\end{tabular}

Table 5. Accuracy check for response surfaces

\begin{tabular}{lccc} 
& Transverse mode & Bending mode & Torsional mode \\
\hline $\mathrm{R}^{2}$ & 0.999 & 0.996 & 0.995 \\
$\mathrm{RMSE}$ & 0.01843 & 0.07969 & 0.13453 \\
\hline
\end{tabular}

\begin{tabular}{|c|c|c|c|c|c|c|}
\hline \multirow{2}{*}{ Name } & \multirow{2}{*}{ Parameter } & \multicolumn{3}{|c|}{ Objective } & & \\
\hline & & Type & & Target & \multicolumn{2}{|l|}{ Type } \\
\hline Seek P7 $=17,556 \mathrm{~Hz}$ & P7 - Translational Mode & Seek Target & $\nabla$ & 17,556 & No Constraint & $\nabla$ \\
\hline Seek P8 $=28,926 \mathrm{~Hz}$ & P8 - Bending Mode & Seek Target & - & 28,926 & No Constraint & $\nabla$ \\
\hline Seek P9 $=41,231 \mathrm{~Hz}$ & P9 - Torsional Mode & Seek Target & - & 41,231 & No Constraint & $\nabla$ \\
\hline
\end{tabular}

Fig. 12. Optimization objective values

As a result of optimization, the changes in modulus of elasticity of the components before and after the calibration process are represented in Table 6. It can be realized that the modulus of elasticity of the components used in the FE model changed by $3 \%-\% 42$. Also, the comparison between the natural frequency values identified by experimentally and numerically is indicated in Table 7. Differences between numerical and experimental natural frequencies corresponding to the transverse, bending and torsional modes vary between $0.46 \%$ and $2.33 \%$. Therefore, it is seen that the FE model obtained as a result of the calibration process reflects the dynamic behavior of the historical masonry bridge in a more realistic way.

\section{Conclusion}

This study deals with the determination of modal parameters of the historical masonry bridge by using OMA. For this purpose, Sarpdere Historical Bridge located in Ordu was chosen for the experimental study, FE modeling and FE model calibration. 
Table 6. Differences in modulus of elasticity between before and after calibration

\begin{tabular}{lccc}
\hline $\begin{array}{l}\text { Component } \\
\text { No }\end{array}$ & $\begin{array}{c}\text { Modulus of elasticity } \\
\text { before calibration (MPa) }\end{array}$ & $\begin{array}{c}\text { Modulus of elasticity } \\
\text { after calibration (MPa) }\end{array}$ & Error (\%) \\
\hline 1 & $1 \times 10^{9}$ & $1 \times 10^{9}$ & 0 \\
2 & 4000 & 5170 & 29.25 \\
3 & 2000 & 1292 & -35.4 \\
4 & 2000 & 1188 & -40.6 \\
5 & $1 \times 10^{9}$ & $1 \times 10^{9}$ & 0 \\
6 & 4000 & 4122 & 3.05 \\
7 & 4000 & 2682 & -32.95 \\
8 & 2000 & 1164 & -41.8 \\
\hline
\end{tabular}

Table 7. Differences in natural frequencies between before and after calibration

\begin{tabular}{lccccc}
\hline Mode & $\begin{array}{c}\text { Experimental } \\
(\mathrm{Hz})\end{array}$ & $\begin{array}{c}\text { FE model } \\
\text { before calibration }(\mathrm{Hz})\end{array}$ & $\begin{array}{c}\text { Error } \\
(\%)\end{array}$ & $\begin{array}{c}\text { FE model after } \\
\text { calibration }(\mathrm{Hz})\end{array}$ & $\begin{array}{c}\text { Error } \\
(\%)\end{array}$ \\
\hline Transverse & 17.56 & 26.22 & 49.3 & 17.97 & 2.33 \\
Bending & 28.93 & 30.66 & 6.0 & 28.35 & -2.00 \\
Torsional & 41.23 & 42.86 & 3.9 & 41.42 & -0.46 \\
\hline
\end{tabular}

The ambient vibration test was carried out under environmental conditions such as traffic movements, human walking and wind loads, etc. The EFDD and SSI methods were implemented to identify the natural frequencies, mode shapes, and damping ratios experimentally. ANSYS software was used to construct the historical masonry bridge model and determine the modal parameters of the FE model numerically. The following conclusions can be drawn from this study:

- The natural frequencies of the historical masonry bridge were experimentally identified as $17.56 \mathrm{~Hz}, 28.93 \mathrm{~Hz}, 41.23 \mathrm{~Hz}$, and $17.55 \mathrm{~Hz}$, $28.90 \mathrm{~Hz}, 41.69 \mathrm{~Hz}$ for EFDD and SSI techniques, respectively. As can be seen, there is a good harmony between natural frequencies of the historical masonry bridge obtained by EFDD and SSI methods.

- The bridge mode shapes appear as transverse, bending and torsional mode by using both techniques.

- The MAC values of the mode shapes obtained in both EFDD and SSI methods were calculated as $0.999,0.911$ and 0.923 for transverse, bending and torsional modes, respectively.

- The natural frequencies were extracted from the initial FE analysis as $26.216 \mathrm{~Hz}, 30.656 \mathrm{~Hz}$, and $42.857 \mathrm{~Hz}$ for transverse, bending and torsional mode shapes, respectively. The maximum difference was determined as $49.3 \%$ for the transverse mode between the experimentally and numerically calculated natural frequencies.

- The RS-based FE model calibration is carried out to eliminate residuals between experimental and numerical modal parameters. Natural frequencies identified experimentally are chosen as objective values in the optimization procedure. The maximum differences were reduced from $49.3 \%$ to $2.33 \%$.

In light of all results obtained in the study, the initial FE model of the structures is not sufficient to represent their dynamic behavior. Therefore, the initial FE model should be calibrated by considering the changes in parameters such as material properties and boundary conditions. Thus, a more realistic FE model can be created which can 
exhibit the dynamic behavior of engineering structures. Furthermore, it can be seen that the RS method allows to further improve the correlation between experimental and analytical modal parameters, therefore, it is an effective approach in the FE model calibration process.

\section{Declaration of conflicting interests}

The author(s) declared no potential conflicts of interest with respect to the research, authorship, and/or publication of this article.

\section{Acknowledgments}

This study is based on the part of the doctoral dissertation done by Emre Alpaslan under the supervision of Prof. Dr. Zeki Karaca and is supported by Ondokuz Mayis University as PYO.MUH.1904.17.009 Scientific Research Project.

\section{References}

[1] Sevim B, Altunışık AC, Bayraktar A (2012) Earthquake behavior of berke arch dam using ambient vibration test results. Journal of Performance of Constructed Facilities 26: 780-792.

[2] Rathi AK, Chakraborty A. Adaptive response surface-based FE model updating for operational modal analysis of RC road bridge. 4th Optimization and Stochastic Days, 18-19 September 2014, Bangolore, India.

[3] Deshan S, Qiao L, Inamullah K, Xiaohang Z (2015) A novel finite element model updating method based on substructure and response surface model. Engineering Structures 103: 147-156.

[4] Zong Z, Lin X, Niu J (2015) Finite element model validation of bridge based on structural health monitoring-Part I: Response surface-based finite element model updating. Journal of Traffic and Transportation Engineering (English Edition) 2: 258-278.

[5] Başaran H, Demir A, Ercan E, Nohutçu H, Hökelekli E, Kozanoğlu C (2016) Investigation of seismic safety of a masonry minaret using its dynamic characteristics. Earthquakes and Structures 10: 523-538.

[6] Günaydın M, Adanur S, Altunışık AC, Sevim B, Bayraktar A (2017) Finite modeling updating effects on the dynamic response of building models. Journal of Testing and Evaluation 45: 1630-1649.

[7] Alpaslan E. Damage detection of historical masonry bridges with analytical model and experimental techniques. PhD Thesis. Ondokuz Mayıs University, Samsun, Tukey, 2019.

[8] Ministry of Transport and Infrastructures, Department of Structures, Division of Historical Bridges. Report and Restoration Project of the Historical Sarpdere Bridge, Samsun, Turkey. Y11, sayı vs bilgiler eksik

[9] Onat O, Yön B (2018) Adopted material properties of historical masonry structures for finite element models: Mosques and bridges, Turkish Journal of Science and Technology 13: 69-75.

[10] Brincker R, Zhang L. Frequency domain decomposition revisited. 3rd International Operational Modal Analysis Conference, 4-6 May 2009, Portonovo, Italy.

[11] Bendat JS, Piersol AG. Random Data: Analysis and Measurement Procedures. John Wiley and Sons, New York, 2010.

[12] Brincker R, Zhang L, Andersen P. Modal identification from ambient response using frequency domain decomposition. Procededing of IMAC-XVIII: A conference and exposition on structural dynamics, 7 February 2000, Texas, USA.

[13] Van Overschee P, De Moor BL. Subspace Identification for Linear System: TheoryImplementation-Applications. Springer, USA, 1996.

[14] Hermans L, Der Aueveraer V (1999) Modal testing and analysis of structures under operational conditions: Industrial applications. Mechanical Systems and Signal Processing 13: 193-216.

[15] Reynders E, De Roeck G (2008) Reference-based combined deterministic-stochastic subspace identification for experimental and operational modal analysis. Mechanical System and Signal Processing 22: 617-637.

[16] Pastor M, Binda M, Harčarik T (2012) Modal assurance criterion. Procedia Engineering 4: 543548.

[17] Umar SB. Response surface methodology for damage detection using frequency and mode shapes. MSc Thesis. University of Technology, Malaysia, 2015.

[18] Cheng J, Zhang J, Cai CS, Xiao RC (2007) A new approach for solving inverse reliability problems with implicit response functions. Engineering Structures 29: 71-79. 
[19] Landman D, Simpson J, Vicroy D, Parker P (2007) Response surface methods for efficient complex aircraft configuration aerodynamic characterization. Journal of Aircraft 44: 1189-1195

[20] ANSYS Workbench (2013) Ansys Incorporation, Pennsylvania.

[21] Box GEB, Draper NR. Empirical Model-Building and Response Surfaces. John Wiley and Sons, New York, 1987.

[22] Fand SE, Perera R (2009) A response surface methodology based damage identification technique. Smart Material and Structures 18: 065009 .
[23] ARTeMIS Modal 1.5 (2012) Structural Vibration Solution, Denmark.

[24] Onat O (2019) Fundamental vibration frequency prediction of historical masonry bridges. Structural Engineering and Mechanics 69: 155-162.

[25] Deere DV, Miller RL. Engineering classification and index properties of Intact Rock. Department of Civil Engineering, University of Illinois, Urbana, Technical Report, No: AFWL-TR-65-116, 1966, pp. 327. 